\title{
X-RAY SPECTRA OF TOKAMAK PLASMA
}

\author{
B.S. Fraenkel \\ Dept. of Physics, the Hebrew University, Jerusalem, Israel
}

A spectrum covering Fe XXV to Fe XXII $x$ ray lines from a tokamak (PLT) plasma has been obtained. Results indicate similarity with the flare spectrum in this region - the forbidden lines also appear in the PLT śpectrum.

$T_{e}$ was about $1.8 \mathrm{keV}$ - a value confirmed by intensity of dielectronic recombination lines. $T_{i}$ was found to be not more than $0.7 \mathrm{keV}$, by Doppler width of the He like singlet $1 s^{2}-1 s 2 p{ }^{1} S_{0}-{ }^{1} p^{0}$. A Johansson crystal was used, with the spectrograph adapted for an extended X-ray source. 